Case Report

\title{
A Case Presentation on Seizure Disorder (Epilepsy during Pregnancy)
}

\author{
Shehataz ${ }^{1}$, A. Jeyasudha ${ }^{2}$, B. Sree Renjini ${ }^{3}$, Sanjana S. ${ }^{4}$ \\ ${ }^{1}$ UG student, ${ }^{2}$ Principal, ${ }^{3} \mathrm{HOD},{ }^{4}$ Asst.Lecturer, OBG Nursing department, PSG CON. CBE, 641 004, Tamilnadu, India. \\ *Corresponding Author : Sanjana S., Asst. Lecturer, OBG Nursing department, PSG CON. CBE, 641 004, Tamilnadu, India. \\ Mobile : +91 76393 73260, E-mail : sanjanasiby2011@gmail.com.
}

Received

: 29.08.2017

Review Completed : 27.12.2017

Accepted

: 05.01.2018

Keywords : seizure, epilepsy, eclampsia, status epilepticus, pregnancy

\begin{tabular}{|c|}
\hline Access this article online \\
\hline Quick Response Code \\
\hline
\end{tabular}

\begin{abstract}
Seizures during pregnancy complicate $<1 \%$ of all gestations; however, they are associated with increased adverse maternal and perinatal outcomes (acute and long term). The differential diagnosis of seizures in pregnancy is extensive. Determining the underlying etiology is crucial in the management of these patients. Medical providers caring for pregnant women should be educated about possible etiologies of seizures during pregnancy and the importance of prompt management of these women in a timely fashion. Evaluation and management should be performed in a stepwise fashion and may require a multidisciplinary approach with other specialtiessuch asneurology.
\end{abstract}

Objective : The objective of this article is to increase awareness and to provide a stepwise approach toward the diagnosis and management of pregnancies complicated by seizures.

\section{Introduction}

Epilepsy is the most commonly encountered serious neurological problem in obstetrical practice. The incidence of epilepsy in pregnancy is $0.3-0.5 \%$ in different populations around the world. The proportion of the population on antiepileptic drugs (AEDs) in pregnancy ranges from 4-9 per 1000 people. The disease and these medications may have significant impact on reproductive function, contraceptive choice and efficacy. During pregnancy, the obstetricians, neurologist, primary care physicians and nurses have the unenviable task of ensuring freedom from seizures during pregnancy while minimizing possible adverse effects of AEDs on the fetus and maximizing the opportunity for a good outcome for the mother and the newborn. The effect of epilepsy on pregnancy is uncertain. Frequency of convulsion is unchanged in majority $(50 \%)$ and is increased in some Oestrogen probably activate seizure foci. There is increased plasma clearance of anticonvulsant drugs during pregnancy. The adverse effects on the mother are third trimester bleeding and megaloblastic anemia-may be related to anticonvulsant induced folate deficiency. The purpose of this case presentation is to provide an update on management of women with epilepsy before and during pregnancy, and in the postpartum period, paying particular attention to the areas where nursing care is involved.

\section{Major effects of epilepsy on pregnancy}

Incidence of fetal malformations, IUGR, Oligohydraminos and Still births is increased. Birth defects are increased by two fold. This can be due to the hazards of anticonvulsants used. All the drugs as implicated, maximum with phenytoin and least with carbamazepine. The malformations include cleft lip or palate, mental retardation, cardiac abnormalities, limb defectsand hypoplasia of the terminal phalanges. Sodium Valporate is associated with neural tube defects. There is chance of neonatal hamorrhage and is related to anticonvulsant induced vitamin $\mathrm{k}$ dependent 
coagulopathy. The risk of developing epilepsy to the offspring of an epileptic mother is four fold.

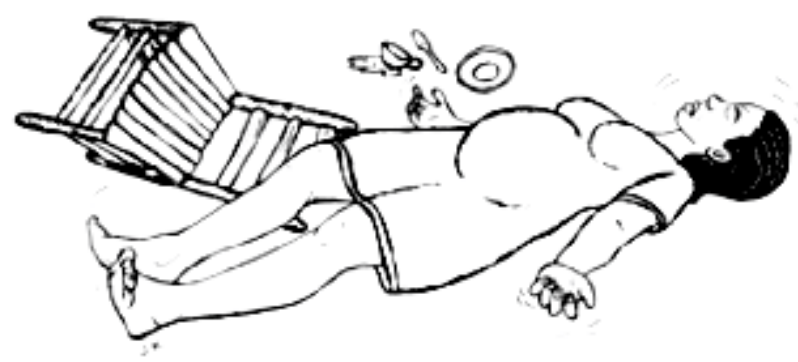

Preconception counseling includes:-

1) To initiate monotherapy (if possible) replacing polytherapy.

2) To administer folic acid 1mg daily.

3) Importance of prenatal diagnosis is discussed.

\section{Management}

The dose of the chosen drug should be kept as low as possible and to be monitored regularly from the serum level. The commonly used drugs are: Phenobarbitone 60 $180 \mathrm{mg}$ daily in divided doses. Phenytoin $150-300 \mathrm{mg}$ daily in two divided doses, carbamazepine 0.8-1.2gm daily in divided doses. The fits are controlled by intravenous diazepam 10-20mg Folic acid 1mg daily to be continued throughout pregnancy. Prenatal diagnosis with serum a FP at 16 week and detailed fetal anatomy scan at 18 weeks with real time Ultrasonography is done. There is decreased in free level of most of the anticonvulsants in pregnancy. This is due to reduced absorption and at the same time increased hepatic metabolism and renal clearance. Vitamin $\mathrm{k} 10 \mathrm{mg}$ a day orally is to be given in the last two weeks. Termination of pregnancy may have to be considered in consultation with neurologist.

There is no contraindication for breast feeding .The infant may be drowsy. Readjustment of the anticonvulsant dosage is necessary and to bring down the dose to the pre pregnant level by 4-6weeks postpartum. Steroidal contraceptives are better to be avoided due to Hepatic microsomal enzyme induction.

\section{Case Description}

Present Medical History
A 28yrs primi gravid women at 35 weeks +3 days, $\mathrm{k} / \mathrm{c} / 0$ Arachnoids cyst has referred to PSG hospitals from a local hospital for recurrent seizure .On arrival she had an on episode of seizures, drowsy, not responding to commands, BP $90 / 60 \mathrm{mmHg}, \mathrm{SPO} 2$ less than $90 \%$ in RA,TEM P-105 F,GRBS-248MG/DL, pupils reactive. There were no complaints of vomiting and neck stiffness. No h/o Vomiting, neck stiffness etc. On ultrasound examination the estimated weight of the fetus was $2.0 \mathrm{~kg}$, FHR$148 \mathrm{bt} / \mathrm{min}$ and fetal condition was good. Medical opinion and Neuro opinion was sought and patient was stabilized with anti epileptics and sedatives. She was intubated and emergency LSCS in view of eclampsia/ status epileptics was done on 1/5/17 and delivered a boy baby of $2.3 \mathrm{~kg}$ at 03.04am who cried immediately with the APGAR score IMIN 7/10 and 5MIN 8/10.Inj.Vit $K$ was administered resuscitation was done. Baby was kept in NICU for one day observation, expressed breastfeed was initiated later on baby was made to fed on the M other's breast.

\section{Past Medical and Surgical History}

K/C/O ARACHNOID CYST diagnosed on 13/06/2005 following a seizure attack, on CT scan report an extra axial cystic lesion noted in the Rt fronto parietal region possibly arachnoid cyst and immediately operated and shunting was done 11 yrs ago. Pt was on TAB.ZEPTOL CR 300M G till Dec 2016

\section{Present Pre and Post Op History}

M edications administrated during hospitalization was Inj. Atracurium 25mg Iv Stat, Inj. Dexa 4mg Iv Stat, Inj. Fentanyl 100mic Iv Stat, Inj. Lorazepam 1mg, Inj, M idaz $2 \mathrm{mg}$ Iv. Inj Perfalgan Bd, Inj Levipill 500mg1-0-1, Zonac Suppository10-1,Inj Tramadol50mg SOS, Inj Keppra 500mg. T.Zeptol Cr 300mg 1-0-1,T. Hifinac 1-0-1.C.Lactare 2-22,Autrin,Macalvit $500 \mathrm{mg}$ 0-1-0.Post operatively MRI BRAIN+M RV was done on 1.5.17 showed arachnoids cyst in RT frontal region increased in size. VP shunt insitu, volume loss in RT parieto temporal lobes with prominent sylvan fissure, diffuse pachymeningeal enhancement along with cerebral convexities. Venogram showed hypo plastic right transverse sinus. Post op period was uneventful. 
Neurology opinion was sought and was asked to continue T.ZEPTOL CR 300M G 1-0-1 1/2 x 1month. M other improved symptomatically and hence being discharged with following advice.

Exclusive breastfeeding

Cu-Tat 6 Weeks.

T. Zeptol Cr 300mg 1-0-1 1/2M onth

T. Hifinac 1 Sos

T. Autrin 0-0-1 $1 \times 3$ months

T. M acalvit 500mg 0-1-0 6 months

Nursing Diagnosis

\section{Risk for Injury related to seizure activity}

Nursing Goal: Preventing Injury to mother and fetus.

Nursing Interventions

- Provide a safe environment by padding side rails and removing clutter which may be harmful to the mother.

- Monitor compliance in taking anti-seizure medications to determine risk for seizure.

- Keep suction, Ambu bag, mouth piece at the bedside to maintain airway and oxygenation if needed.

- Place the bed in a low position.

- Do not put anything in the mother's mouth during a seizure.

- Place the mother on side during a seizure to prevent aspiration.

- Protect the mother's head during a seizure. If seizure occurs while ambulating or from chair, cradle head or provide cushion/support for protection against head injury.

- Stay with the mother who is ambulating or who is in a confused state during seizure.

- Manage with medications (drugs which are allowed to take during pregnancy as physician order).

\section{Ineffective Tissue Perfusion (cerebral) related to seizure activity}

Nursing Goal: M aintaining Cerebral Tissue Perfusion

Nursing Interventions

- Maintain a patent airway until mother is fully awake after a seizure.

- Provide oxygen during the seizure if a cyanotic change occurs.

- Stress the importance of taking medications regularly.

- Monitor serum levels for therapeutic range of medications.

- M onitor patient for toxic adverse effects of medications.

- Monitor platelet and liver functions for toxicity due to medications

2. Ineffective breathing pattern related to neuromuscular impairment secondary to prolonged tonic phase of seizure or during postictal period as evidenced by abnormal respiratory rate, rhythm, and/ or depth. Airway management

- Monitor respiratory and oxygenation status to determine presence and extend of problem and to initiate appropriate interventions.

- Position patient (side lying) to maximize ventilation potential.

- Identify patient requiring actual/potential airway insertion to facilitate intubation as necessary.

- Perform endotracheal or nasotracheal suctioning to maintain airway as needed.

- Loosen clothing to prevent restricted breathing.

- Apply oxygen as appropriate to maintain oxygenation and prevent hypoxia.

\section{Knowledge Deficit related to}

- Lack of exposure, unfamiliarity with resources

- Information misinterpretation

- Lack of recall; cognitive limitation.

\section{Intervention}

1. Ascertain level of knowledge, including anticipatory needs to assess readiness to learn.

2. Review pathology and prognosis of condition and lifelong need for treatments as indicated. Discuss patient's particular trigger factors (flashing lights, hyperventilation, loud noises, video games, TV viewing). Provides opportunity to clarify or dispel misconceptions and present condition as something that is manageable within a normal lifestyle.

3. Review possible effects of hormonal changes. Alterations in hormonal levels that occur during 
menstruation and pregnancy may increase risk of seizure.

Discuss significance of maintaining good general health, (adequate diet, rest, moderate exercise, and avoidance of exhaustion, alcohol, caffeine, and stimulant drugs). Regularity and moderation in activities may aid in reducing or controlling precipitating factors, enhancing sense of general well-being, and strengthening coping ability and self-esteem.

4. Review medication regimen, necessity of taking drugs as ordered. Lack of cooperation with medication regimen is a leading cause of seizure breakthrough.

\section{Conclusion}

Pregnancy in patients with seizure disorders can be complicated by a variety of maternal and fetal issues. Patients can experience higher rates of seizures because of the lower serum plasma levels of their AEDs. The fetus is likely to be at increased risk for congenital abnormalities, most notably facial clefts, cardiac anomalies, and neural tube defects.

Long-term outcomes show that children of patients with

\section{References}

1. D.C.Dutta.(2004). Textbook of obstetrics including perinatology and contraception $6^{\text {th }}$ edition.new central book Agency.298

2. Laganà AS, Triolo O, D'Amico V, Cartella SM, Sofo V, Salmeri FM, et al. Management of women with epilepsy: from preconception to postpartum. Arch Gynecol Obstet. 2016 M ar. 293 (3):493-503. [M edline].

3. National Institute for Health and Care Excellence. The epilepsies: the diagnosis and management of the epilepsies in adults and children in primary and secondary care. NICE clinical guideline 137. [M anchester]:NICE; 2012

4. Smith PE, Saunders J, Dawson A, Kerr MP. Intractable seizures in pregnancy. Lancet 1999;354:1522 seizure disorders may have lower IQs and higher rates of developmental delay. Syndromes related to several of the AEDs and to specific abnormalities observed in patients with seizure disorders are not known to affect rates of chromosomal abnormalities.

\section{Prognosis}

Women with epilepsy should be treated during pregnancy by a team of providers, including a primatologist and a neurologist that can focus on balancing the risks of seizures versus the administration of AEDs. Pre-conceptual management with tapering to AED monotherapy and foliate supplementation are recommended. During pregnancy, AED levels should be monitored closely, and the fetus should be carefully screened for anomalies with serum testing, Ultrasonography, and, possibly, amniocentesis. Vitamin K supplementation for the patient and then for the newborn at the end of the pregnancy is controversial; the risks and benefits of this aspect of management should be discussed by the entire team of providers caring for the patient. With careful treatment of these patients, more than $90 \%$ have an entirely uncomplicated pregnancy 